\title{
Development of spreadsheet based quantity take-off and cost estimation application
}

\author{
Ö.H. Bettemir ${ }^{*}$ \\ Inonu University, Department of Civil Engineering, Malatya, Turkey
}

\begin{abstract}
Quantity take-off and cost estimation are the most time consuming and costly work items of the project planning phase. A structure consists of significantly many components, and quantities of all of these work items have to be computed as well as necessary workmanship and materials should be determined. If quantity take-off is computed incorrectly, then cost will also be estimated erroneously. Therefore, computation of quantity take-off correctly has vital importance. Each constructed structure is unique, for this reason quantity take-off has to be prepared for every structure. In this study, a spreadsheet application is developed for the computation of quantity take-off with minimum human intervention and endeavor. Positions of the load bearing sections and architectural elements are defined by nodes and dimensions of these elements are identified by entering related information to data cells. Topologies of the structural and architectural elements are formed to determine the neighborhood and intersections. Voids are computed and subtracted to obtain exact quantities. Necessary materials and workmanships are determined by using database and cost is estimated by using unit prices of these items. Quantity take-off and cost is computed fast and accurately with minimized data input. Consequently, design offices can avoid purchasing expensive quantity take-off software by utilizing this approach.
\end{abstract}

\section{Keywords}

Quantity take-off, Automated cost estimation; Spatial conflict detection; Project planning

Received: 01 June 2018; Accepted: 21 September 2018

ISSN: 2630-5771 (online) C 2018 Golden Light Publishing All rights reserved.

\section{Introduction}

Automated quantity take-off by Building Information Model (BIM) is a fast and practical way of preparing quantity take-off (QTO) documents. Both private sector and researchers pay significant attention on the subject. Consequently there are many commercial applications that execute automated quantity take-off. Innovaya Estimating, Vico Estimator, Tokmo Production System, Success Design Exchange, Timberline Extended, and Winest Design Estimation Pro are some of the common BIM-based cost estimation software [1].

Construction sector is eager to implement automated QTO software since it provides important savings in terms of time when compared with traditional quantity take-off methods [2]. In addition to this, automated quantity take-off improves the accuracy of QTO because rounding errors are removed and omissions are lessened [3]. Despite the advantages of BIM on the automated quantity take-off, construction companies implement BIM for visualization at most, then

* Corresponding author

Email: onder.bettemir@inonu.edu.tr 
design and modeling of the architectural model, collision detection and cost estimating [4].

Lee et al. [5] proposed a method to deduce work items without human intervention. Tiling work was examined for a case study. Kim et al. [6] developed an automated modeling method which automatically prepares quantity take-off of the interior parts of the building. Aram et al. [7] developed framework for automated quantity takeoff and cost estimation of precast concrete products with established 3D parametric models. Choi et al. [8] developed an open BIM-based quantity take-off system for schematic estimation of building frames. Chen et al. [9] developed a framework which exports quantity take-off data and computes necessary man.hours with its embedded database. Furthermore, the system provides construction schedule with minimum input.

Many countries have unique standards for quantity take-off, and this is one of the most important difficulties on the automated quantity take-off. Smith [10] states that cost management is disorganized and organizations about cost estimation should work together. Absence of global standards prevents the development of project cost management profession around the world [11]. Monteiro and Martins [12] state that cost estimation is not performed by applying national standard quantity take-off rules. Plebankiewicz et al. [1] proposed a Polish BIM-based cost estimation application with a specialized BIM measurement and a cost estimate system. Similarly Wijayakumar and Jayasena [13] states that hardware, software, and liveware investments are necessary for the shift of the Sri Lankan construction industry to BIMbased quantity take-off. Didkovskaya et al. [14] reported that in Russia cost engineering is implemented by several large corporations which declared the development of cost management systems. The mentioned deficiencies prevent a perfect automation of quantity take-off. Therefore in 2012 the Royal Institution of Chartered Surveyors (RICS) issued new guidelines which are integrally linked with BIM to enable accurate automated quantity take-off $[15,16]$.
Small and medium enterprises are significantly far away from the automated quantity take-off because of the expensive investment requirements and lack of standardization. In this study, a spreadsheet application which can perform automated quantity take-off of excavation, backfill, concreting and formwork is developed in order to solve the mentioned problem. The developed application can detect intersection of columns, beams and slab by performing conflict detection and outputs very precise quantity take-off report. The prepared spreadsheet can be used by small and medium enterprises by only changing the input values of the place and size of the beams, slabs and columns.

Following section of the paper explains the methodology of quantity take-off of construction items, then method of conflict detection is explained in the third section and the spreadsheet application as well as case study is presented in the fourth section. Finally the results are concluded.

\section{Quantity take-off}

In this section quantity take-off of excavation, concreting, and formwork is presented. Unit of excavation is $\mathrm{m}^{3}$ and the quantity is computed by the excavation project. If the excavation is inclined by the four sides of the excavation, a prismoid shape is obtained and its volume is computed by the following formula [17].

$$
V=\frac{1}{6} * h *\left(A_{\text {bottom }}+4 * A_{\text {middle }}+A_{\text {top }}\right)
$$

In Eq. (1), $h$ is the depth of excavation, $A_{\text {bottom }}$ is the projected area at the bottom, $A_{\text {middle }}$ is the projected area at the middle of the excavation, $A_{\text {top }}$ is the projected area at the top of the excavation respectively.

Eq. (1) requires computation of projected area at the bottom, middle and top of the excavation. If $\mathrm{x}_{\mathrm{B}}$ is the $\mathrm{x}$ coordinate of the corner point at the bottom of the excavation, its $\mathrm{x}$ coordinate at the middle elevation is computed as represented in $\mathrm{Eq}$ (2).

$$
X_{M}=X_{B}+\cos (\alpha) * \frac{h}{2}
$$


The ' + ' sign in front of the cosine term in Eq. (2) becomes '-' when computing the opposite side. Similarly Y coordinates of the middle section are computed. Eq. (1) is implemented when the areas of the three sections are determined.

Reinforced concrete sections are usually uniform and their cross sectional areas are constant through their elongation. However, this is not a rule since in situ cast concrete can have any shape. If the columns or beams of the structure are designed as an irregular shape then the volume of the section is computed by using the Eqs. (1) and (2).

Beams are horizontal elements located between two columns. Span of a beam is measured between the corresponding two columns. Concrete of the beam and the columns should be poured at the same time in order to prevent cold joint. Therefore, there is not any formwork at the beginning and end of the beam. As a result, column surface which intersects with a beam also does not have a formwork. The intersection area should be voided when the quantity take-off of formwork for column is prepared. Similar voids also occur at the formworks of beam and slab intersections. Beam formwork should permit concreting of slab and beam at the same time. Therefore, at the intersection of the beam and slab, there should not be a formwork. The amount of formwork at the intersection of beam and slab is voided from the formwork area of beam.

\section{Spatial conflict detection}

Intersections of beam and column, beam and slab, as well as slab and column have to be detected in order to determine voids. In addition to this, excavation of footings may intersect with neighboring excavation of footing if the side surfaces of the excavation are inclined. Aforementioned intersections can be detected by collision detection algorithm. The collision detection is widely implemented in Geographic Information System (GIS) applications for spatial queries and many algorithms exist for the determination of intersection of two polygons.

O'Rourke et al. [18] developed an algorithm for the detection of intersecting convex polygons. Chin and Wang [19] proposed a linear algorithm for the intersection of two planar polygons. The algorithm can determine whether a convex $\mathrm{n}$-gon and a nonconvex m-gon intersect or not in $\mathrm{O}(\mathrm{n}+\mathrm{m})$ operations. Margalit and Knott [20] presented an algorithm for computing the union, intersection or difference of two polygons. The algorithm lowers the space requirements with acceptable worst case time complexity. Time complexity is important when rendering high resolution computer graphics, however it is not important for the detection of voids of the slab and beam sections.

Excavation for the footings can be inclined and the excavation can intersect with the neighboring excavation for the footing. In this case the quantity of the excavation would be computed more than it is. In order to prevent such deficiency, intersection of the top surfaces of the excavation of footings are examined. If an intersection is detected, the elevation of the intersection is computed and the volume of excavation is computed in two parts. The first part is the portion where intersection has not occurred and the latter part contains the intersected excavation areas.

Spatial conflict detection algorithm checks that if $\mathrm{x}$ or $\mathrm{y}$ coordinates of the corner point of the excavation at the bottom becomes smaller than or greater than any of the corner point of the neighboring excavation portion at the top. If this is the case, excavation portions intersected or not is detected by polygon intersection algorithm.

Detection of intersection of column and beam structural elements is relatively easy. The data input requires entering of start and end nodes of each element. Therefore intersections of structural elements are determined by node topology. Sometimes beams and columns may be eccentric from the structural axis therefore amount of intersection may not be always constant. The position of intersection is also detected and the intersecting area is computed.

\section{Spreadsheet application and case study}

In order to present the proposed quantity take-off methodology a spreadsheet application is prepared on LibreOffice Calc freeware. The structural plan of the structure whose quantity take-off is prepared 
is given in Fig. 1. Floor height is 3 meters and the excavation for the footing has a depth of 1.2 meters. The structure contains four columns, four beams and one slab. Each column is supported by a footing. Footings are $40 \mathrm{~cm}$ wider than the corresponding column in $\mathrm{x}$ and $\mathrm{y}$ directions at the bottom of the footing. On the other hand, footings are $20 \mathrm{~cm}$ wider both in $\mathrm{x}$ and $\mathrm{y}$ directions. In addition to this excavation for the footing of the foundations is $40 \mathrm{~cm}$ wider than the corresponding footing in both $\mathrm{x}$ and $\mathrm{y}$ directions. Sides of the excavation are inclined $45^{\circ}$ in order to prevent slope failure.

The structure has two axis in both $\mathrm{x}$ and $\mathrm{y}$ directions. The axes are spanned by 5 meters in $\mathrm{x}$ direction and 4 meter in y direction. Dimensions of the columns at axis- 1 is 40 by 40 while 80 by 40 at axis-2. Geometric data is entered by using the spreadsheet application. Corners of the axes are represented as nodes. The plane of the structure is identified by four nodes. The floor of the structure is identified by two planes the initial plane represents the ground elevation while the latter represents the ceiling of the structure. Therefore, the one storey structure is represented by eight nodes. In Fig. 2 the coordinates of the nodes are entered by using the data cells B3 to D10.

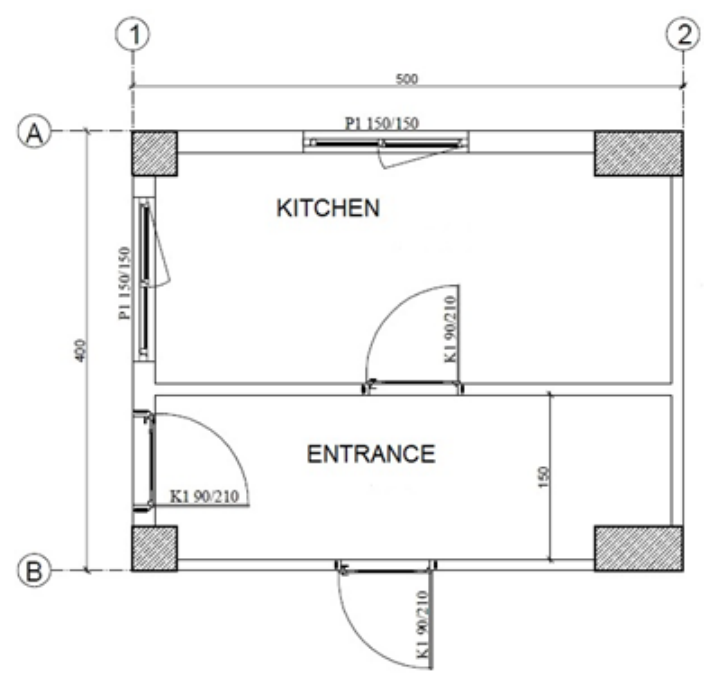

Fig. 1. Architectural drawing of the structure
Spatial data of the columns are entered between the cells B14 to K17. First, start nodes of the column, then width of the column in $x$ and $y$ directions are entered respectively. Eccentricity of the column's bottom left corner with respect to its starting node is entered. This completes the data input for the bottom node of the column. The same information is entered for the top node of the column. This can be recognized as data duplication but it allows computation of quantity take-off of inclined, widening or narrowing columns.

Depth of excavation is entered at line 19. Amount of widening of the footings at the top surface and the elevation of the top surface are entered at line 20. Similar data for the bottom surface of the footings are entered at line 21. Spacing for the workers in the trench is entered at line 23. Slope of the sides of the excavation is given in degree at line 24. Data about the geometry of the beams are entered into the cells B27 to K30. Data related with the beams is very similar to the data related with columns. First, starting node of the beam is entered then width and depth of the column at the starting node is given. Then eccentricity of the beam from the axis is entered in $\mathrm{x}$ and $\mathrm{y}$ directions respectively. This also allows the quantity take-off of beams with not uniform cross sectional area. The same data is entered for the ending node of the beam. Finally, the input data of the slab is given. Position of slab is determined by the nodes of the corners. The complete input data is represented in Fig. 2.

Quantity take-off of construction items are conducted by using their coordinated data. Therefore, coordinates of each structural element are computed. Sample screenshot for the computation of the bottom planar coordinates of the excavation for the footing is represented in Fig. 3. In the spreadsheet formula $x$ coordinate of the start node, and the eccentricity of the column is added. Then enlargement of the footing at the bottom and working space is added and $\mathrm{x}$-coordinate of the footing is computed. 


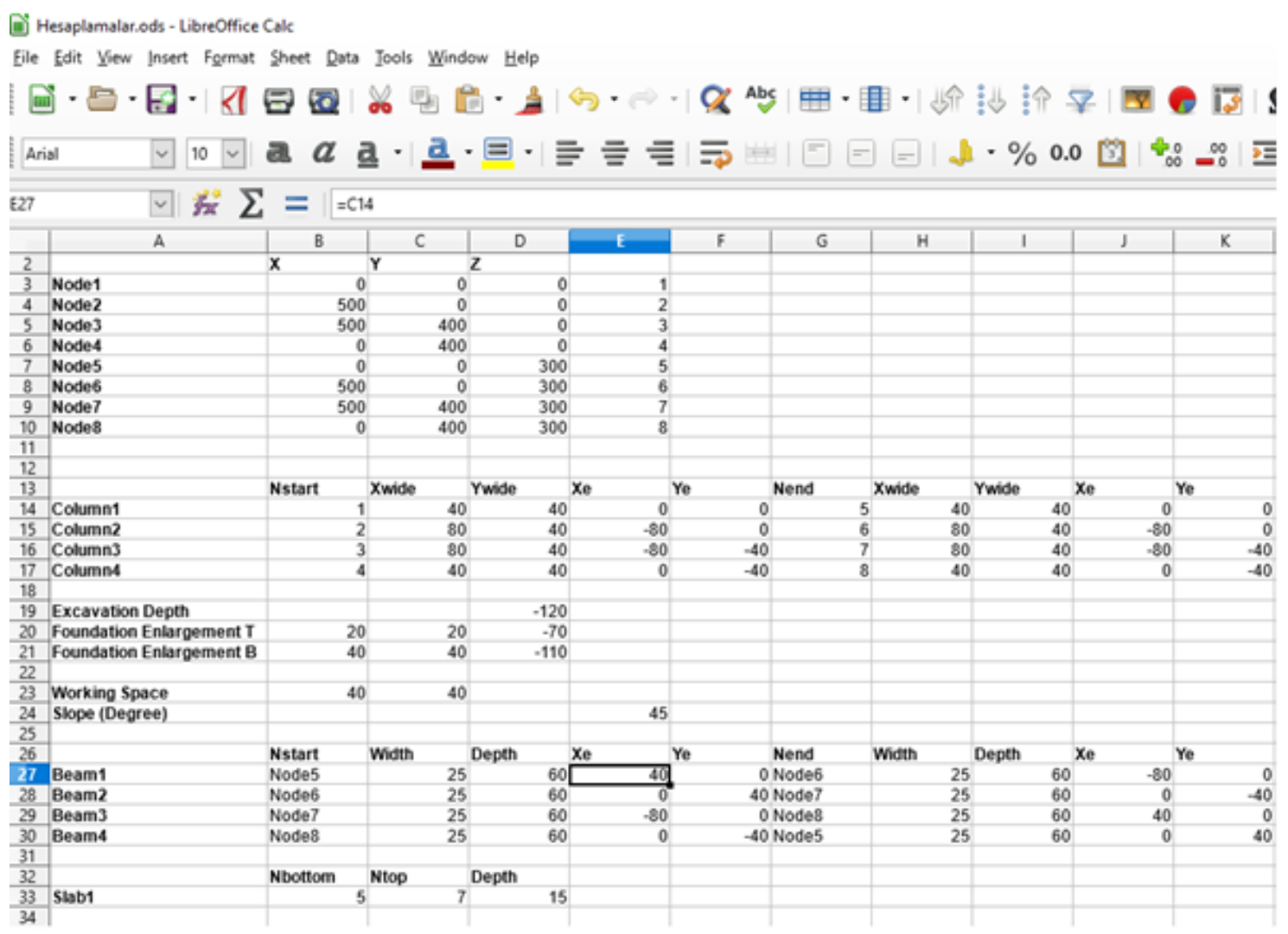

Fig. 2. Data input page of the spreadsheet application

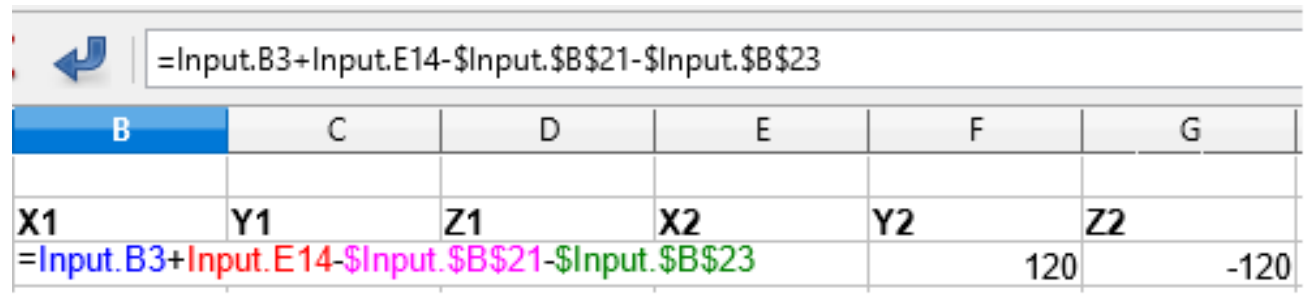

Fig. 3. Computation of the excavation coordinates

Coordinates of the top surface of the excavation is computed by the equation given in Fig. 4. In the equation $B 3$ represents the coordination of the bottom surface. Slope angle is converted to radians from degree and it is multiplied by the depth of the excavation. When computing the opposite side of the slope the "+" sign becomes "-".

Computation of volume of the excavation is performed by calculating three planar surface of the excavation. In Fig. 5 computation steps of the areas of the bottom, middle, and top planar surfaces are

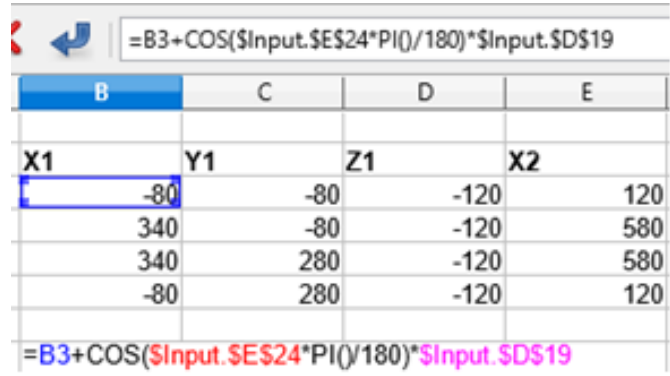

Fig. 4. Determination of the excavation surface 


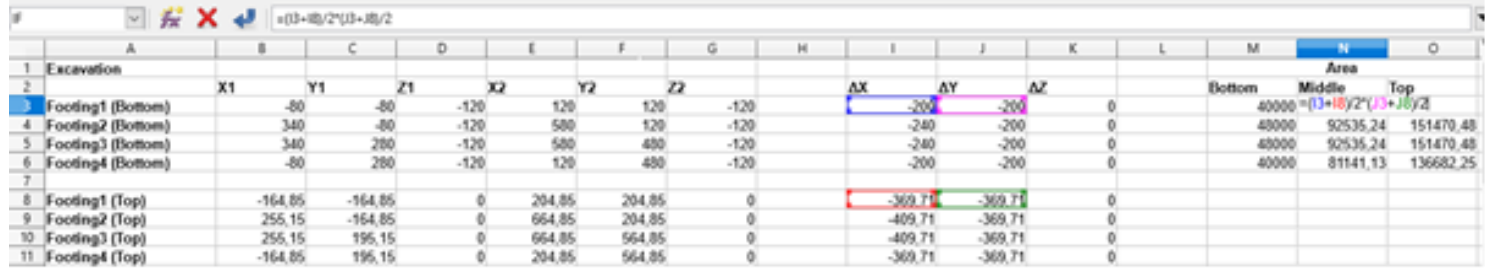

Fig. 5. Computation of the areas of the plane surfaces

represented. First differences in $\mathrm{X}$ and $\mathrm{Y}$ directions are computed by subtracting the coordinate differences between corner nodes. Computed coordinate differences are shown at columns $I$ and $J$ in Fig. 5. Then planar area is computed by multiplying the differences in $\mathrm{x}$ and $\mathrm{y}$ directions. Bottom and top planar areas are computed by multiplying the coordinate differences obtained for the bottom and top nodes respectively. In order to compute the middle planar area, coordinate differences of the top and bottom nodes are averaged and multiplied. The computation is shown in Fig. 5.

Finally, Eq. (1) is implemented and the excavation for the footings is computed. However, when the coordinates of the top surfaces of the excavation is examined it is seen that top surfaces of the excavation for footings 2 and 3 as well as 1 and 4 intersects. Therefore, the computations are incorrect.

Occurrence of a spatial conflict is queried in cell T3 and T4. Height of excavation at the line of intersection is computed in V3 and V4 by the equation represented in Fig. 6. Ratio of the width of the excavated surface and the width of the intersection is computed and this ratio is used for the determination of the height of the excavation at the line of intersection.

Number of excavated portion is equal to the number of footings below the intersection point. However, number of excavated portion becomes half of the number of footing above the intersection point. Therefore in Fig. 7 bottom and intersection coordinates are represented by four lines, while top coordinates are represented by two lines. Computation of planar area is executed in two steps; in the former step which is between lines 8 to
11, planar areas below the intersection point are computed. Then planar areas required for the excavation volume above the intersection point is computed. Computation of second portion of excavation volume is represented between lines 14 and 15. Computation of excavation volume is performed again by utilizing Eq. (1). Volume computations are represented at the columns $A I$ to $A M$ in Fig. 7. Lines 3 to 6 represent excavation volume below the intersection; lines 14 and 15 represent excavation volume above the intersection. Summation of two portions gives the total excavation volume.

If intersection is omitted, volume of excavation is computed as $42.83 \mathrm{~m}^{3}$. On the other hand, if the intersections of excavation portions are taken into account volume of excavation is computed as 42.78 $\mathrm{m}^{3}$. The latter value is the correct measurement. Fig. 8 illustrates the collision of sloped excavation sections. The hatched area is computed twice which is the cause of the aforementioned volume difference of the excavation. The spatial collision detection algorithm reduces the duplication and presents more accurate quantity take-off.

Quantity of concrete for the footings and beams are computed by using Eqs. (1) and (2). Computation procedure is represented between lines 25 to 33 in Fig. 9. Lines between 25 and 28 represent the bottom left corner of the footings. Lines 30 to 33 represent the coordinates of top right corner of the footings. Planar areas are represented at columns $M, N$, and $O$. Concrete volume of each footing is given at column $Q$ between lines 25 to 28 . Total concrete volume for the construction of footings is represented at column $R$ line 28 . 


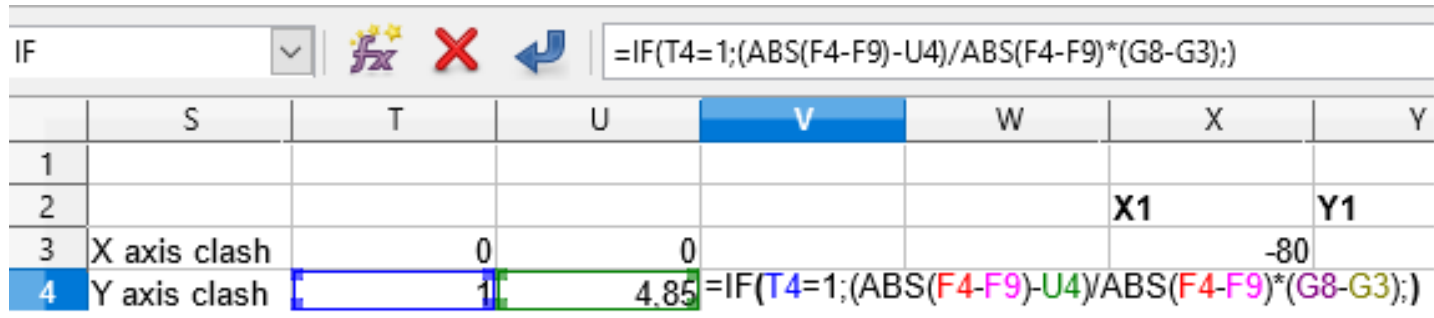

Fig. 6. Determination of the conflict and clashes

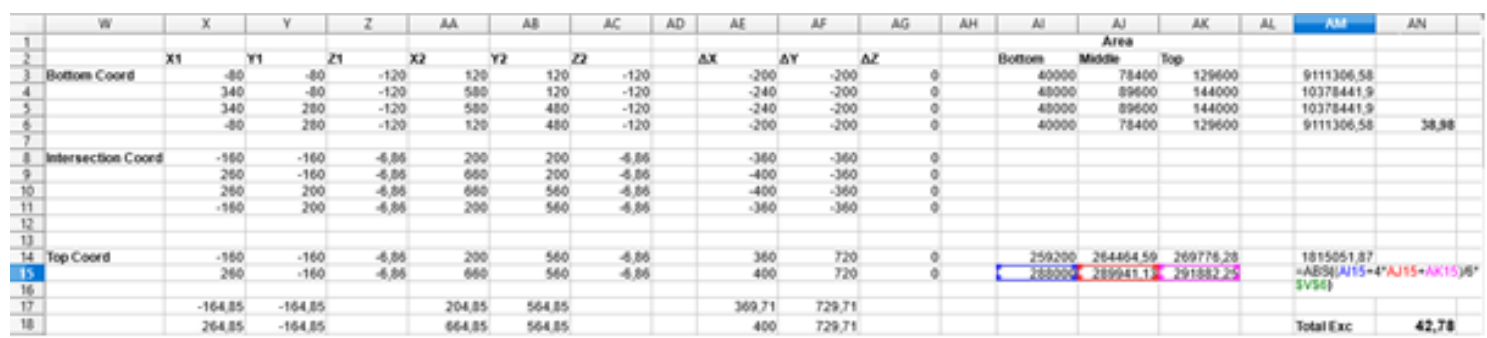

Fig. 7. Volume computation in case of intersection

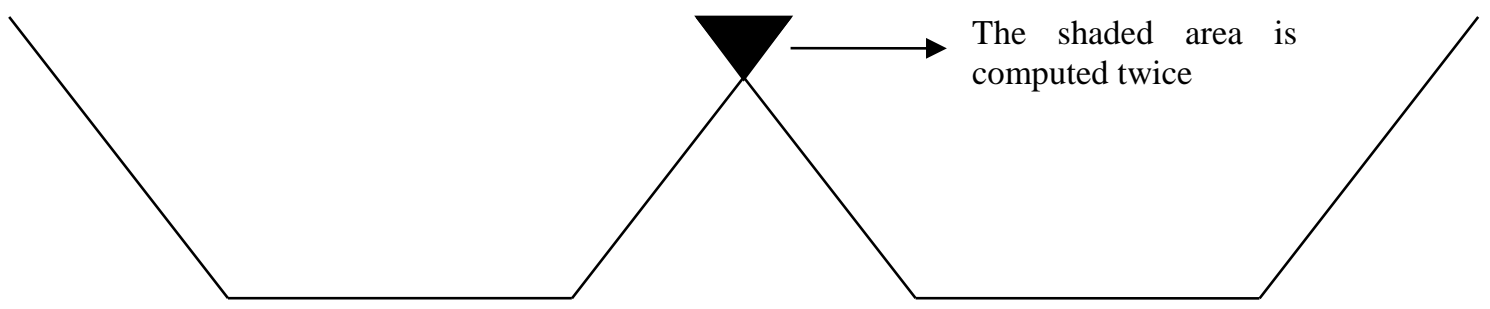

Fig. 8. Intersection of sloped excavation sections

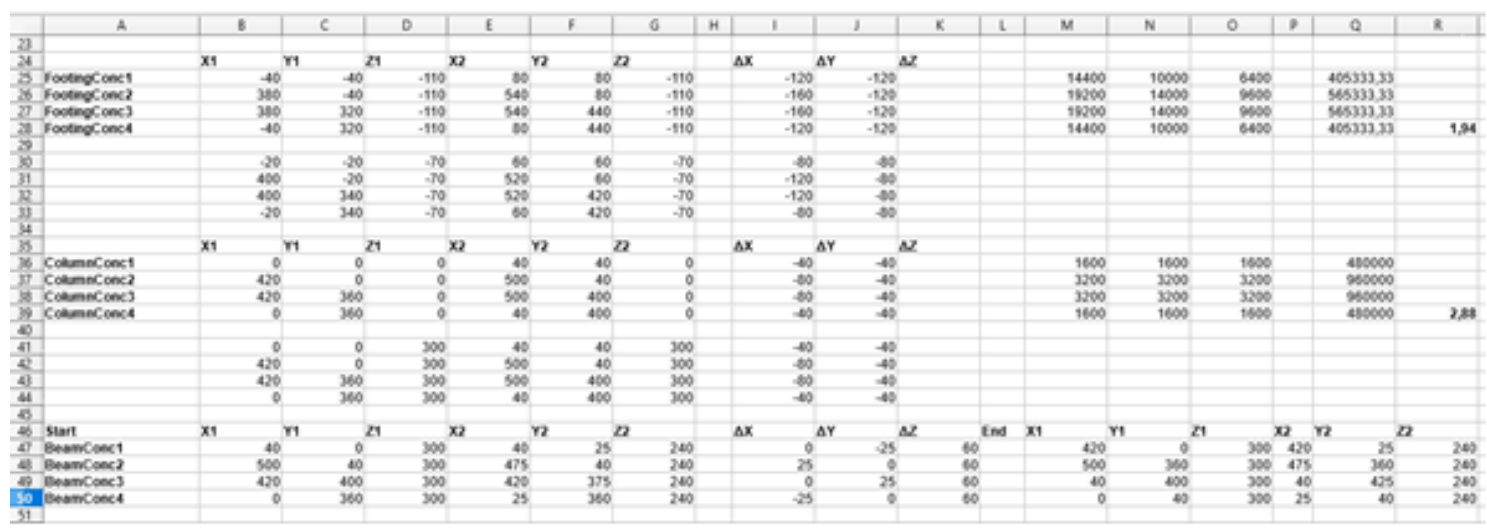

Fig. 9. Computation of quantity of concreting 
Volume of concrete for the columns is represented in Fig. 9 between lines 36 to 39. In the example problem beams are rectangular prism so that their cross sectional area is constant. However, the developed application can also compute the concrete volume of prismoid columns. Column height and cross sectional area of the column is required for the volume computation. Columns extend along their start node to end node. Beams end when they reach a column. By using this assumption, heights of the columns and lengths of the beams are computed. Height of a column is equal to the elevation difference between the elevations of end node and start node. Span of a beam is the distance between the surfaces of columns located at the start and end of the beam. The column surfaces are determined by spatial collision detection.

Spatial data of beams are given in the input tab between lines 27 and 30. Detection of the start and end of a beam is performed by using the spatial data of beam. VLOOKUP command is used in Libreoffice Calc application as shown in Fig. 10. First terms of the equation detects the $\mathrm{X}$ coordinate of the starting node of the beam. Input.B27 is the starting node of the beam, this string is searched through the cells $A 3$ to $D 10$ at the Input tab. Last term 2 is the column where the information would be retrieved. In this case $\mathrm{x}$ coordinate which is represented at the second column is retrieved.

Second term in the equation adds or subtracts the eccentricity of the beam. This term is equal to the width of the column at the corresponding node. If coordinate of starting node is greater than the end node, the eccentricity is subtracted. This is because the portion of the column should be subtracted from the boundary node. The boundary node is at the right of the other boundary node since its $x$ coordinate is greater. The eccentricity is subtracted and the node is translated to the left side. The implemented procedure reverses if the coordinate of the corresponding node is less than the other. If the coordinates are equal, this means that the span of the beam is perpendicular to the examined direction.
Formwork is computed by discretely handling each surface of the columns. Start and end coordinates of each column is computed in lines between 70 and 73 (Fig. 11). Width of the column surface is computed by using the detected coordinates and the computed widths of the column surfaces are reported between the lines 75 to 78 . Areas of the surfaces are computed between the lines 80 to 83 . At this step intersection with slab or beam is checked. If there is an intersection, value 1 is assigned to Bool Int column and the corresponding void is computed.

\section{Discussion of results}

Quantity take-off requires attention, patience, and knowledge. Ignorance of an intersection or collision makes the quantity take-off computations wrong. In addition to this, there are many sections and intersections to take into account. Therefore, preparation of quantity take-off takes significant amount of time. BIM and auxiliary software can perform automated quantity take-off and prepare bid of quantity tables. However, obtaining the software and training their staff requires significant investment and education costs. Therefore small and medium sized construction firms do not usually make investment on automated quantity take-off and prepare their bid of quantity tables manually.

In this study, a spreadsheet application which automatically computes quantity take-off of excavation, concrete, and formwork items is prepared. The data of the structure is input by using the corresponding cells. Dimensions and positions of the structural elements are computed by the linked formulations of the spreadsheet table. Collision and intersections are queried and voids due to intersections are computed. The spreadsheet application significantly shortens the process of preparation of quantity take-off. In addition to this the precision of the final data is more accurate than hand calculations. The spreadsheet application is prepared on a freeware and the small and medium sized construction companies can also implement the defined formulations. 


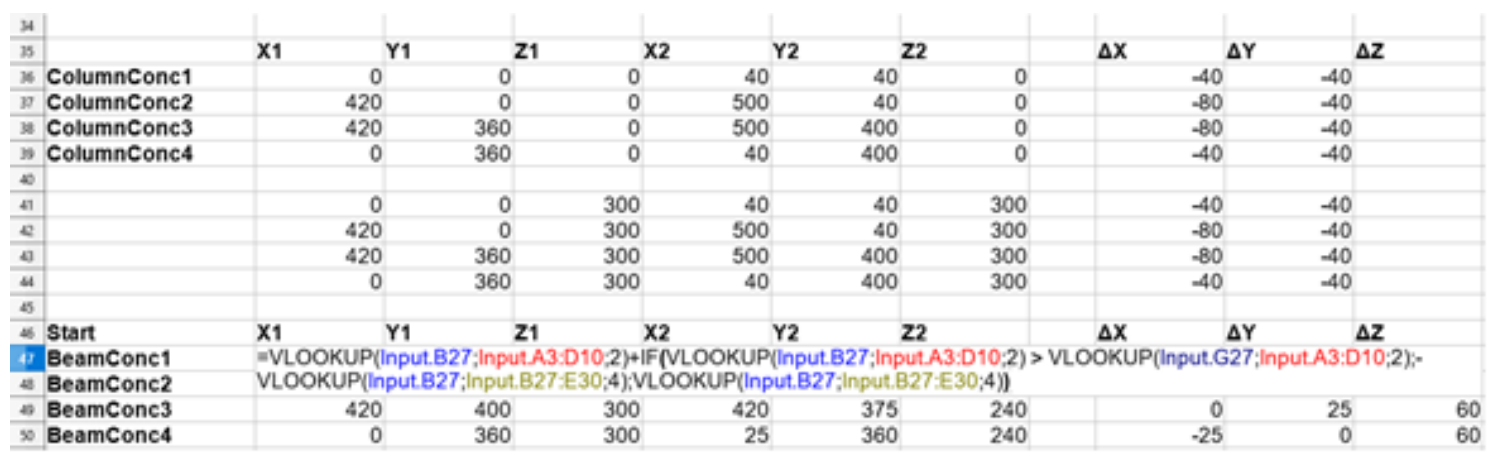

Fig. 10. Detection of start and end of beams

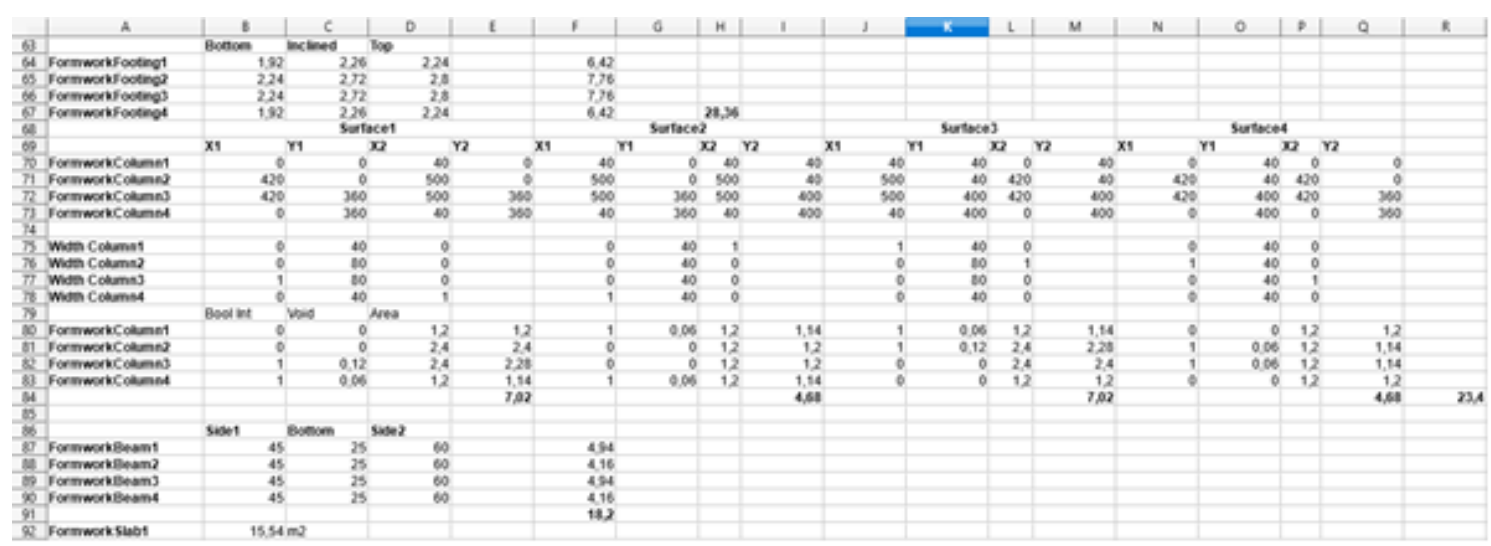

Fig. 11. Computation of quantity of formwork

Quantity take-off of excavation, formwork and concreting tasks are precisely executed by the proposed method. However, this study is not completed. Quantity take-off of reinforcements, plastering, floor covering, painting, door and window frames and other construction items can be added. Quantity take-off of reinforcement requires very detailed information such as clear cover, spacing between reinforcement bars, and diameter of the reinforcement. Entering the aforementioned data manually is an error prone task. Therefore the proposed approach should have data extraction capability from structural design software. These missing features can be added to the developed application as a future study.

\section{Acknowledgments}

This study is supported by the Inonu University Scientific Research Projects Coordination with project number FHD-2017-605.

\section{References}

[1] Plebankiewicz E., Zima K., Skibniewski M. Analysis of the First Polish BIM-based Cost Estimation Application. Procedia Engineering 123 (2015) 405-414.

[2] Bečvarovská R., Matějka P. Comparative Analysis of Creating Traditional Quantity Takeoff Method and Using A BIM Tool. In Construction Maeconomics Conference (2014).

[3] Olsen D., Taylor J. M. Quantity Take-Off Using Building Information Modeling (BIM), and Its Limiting Factors. Procedia Engineering 196 (2017) 1098-1105. 
[4] Sattineni A., Bradford R. H. Estimating with BIM: A survey of US construction companies. Proceedings of the 28th ISARC, Seoul, Korea (2011) 564-569.

[5] Lee S. K., Kim K. R., Yu J. H. BIM and OntologyBased Approach for Building Cost Estimation. Automation in Construction 41 (2014) 96-105.

[6] Kim S. A., Chin S., Yoon S. W., Shin T. H., Kim Y. S., Choi C. Automated Building Information Modeling System for Building Interior to Improve Productivity of BIM-Based Quantity Take-Off. In Proceedings for the 26th International Symposium on Automation and Robotics in Construction (2009) 492-496.

[7] Aram S., Eastman C., Sacks R. A KnowledgeBased Framework for Quantity Takeoff and Cost Estimation in The AEC Industry Using BIM. In ISARC. Proceedings of the International Symposium on Automation and Robotics in Construction (2014).

[8] Choi J., Kim H., Kim I. Open BIM-based Quantity Take-Off System for Schematic Estimation of Building Frame in Early Design Stage. Journal of Computational Design and Engineering 2(1) (2015) 16-25.

[9] Chen S. M., Chen P. H., Chang L. M. A framework For an Automated and Integrated Project Scheduling and Management System. Automation in Construction 35 (2013) 89-110.

[10] Smith P. Project Cost Management-Global Issues and Challenges. Procedia-Social and Behavioral Sciences 119 (2014) 485-494.

[11] Smith P. Global Professional Standards for Project Cost Management. Procedia-Social and Behavioral Sciences 226 (20169 124-131.

[12] Monteiro A., Martins J. P. A survey On Modeling Guidelines for Quantity Takeoff-Oriented BIMBased Design. Automation in Construction 35 (2013) 238-253.

[13] Wijayakumar M., Jayasena H. S. Automation of BIM Quantity Take-Off to Suit QS's Requirements. In Second World Construction Symposium (2013).

[14] Didkovskaya O. V., Mamayeva O. A., Ilyina M. V. Development of cost engineering system in construction. Procedia Engineering 153 (2016) 131-135.

[15] Wu S., Wood G., Ginige K., Jong S. W. A Technical Review of BIM Based Cost Estimating in UK Quantity Surveying Practice, Standards and Tools. Journal of Information Technology in Construction 19 (2014) 534-562.
[16] Smith P. Project Cost Management with 5D BIM. Procedia-Social and Behavioral Sciences 226 (2016) 193-200.

[17] Chapra S. C., Canale R. P. (1998). Numerical methods for engineers, New York: McGraw-Hill (1998).

[18] O'Rourke J., Chien C. B., Olson T., Naddor D. A New Linear Algorithm for Intersecting Convex Polygons. Computer Graphics and Image Processing, 19(4) (1982) 384-391.

[19] Chin F., Wang C. A. Optimal Algorithms for The Intersection and The Minimum Distance Problems Between Planar Polygons. IEEE Transactions on Computers 32(12) (1983) 1203-1207.

[20] Margalit A., Knott G. D. An Algorithm for Computing the Union, Intersection or Difference of Two Polygons. Computers \& Graphics 13(2) (1989) 167-183. 